\title{
COVID-19 pandemic restrictions and problems faced by patients due to difficult hearing.
}

\author{
${ }^{1}$ Umesh Jayarajah, ${ }^{1}$ Oshan Basnayaka, ${ }^{1}$ Heshan Siriwardena, ${ }^{1}$ Anuruddha M Abeygunasekera \\ ${ }^{1}$ Colombo South Teaching Hospital, Kalubowila, Sri Lanka.
}

\begin{abstract}
:
Aims: The impact of COVID-19 is considerable in patients with hearing impairment who are faced with difficulties in communication due to masks and social distancing. We aimed to provide a preliminary description about difficulties faced by a group of patients due to hearing impairment. We collected data from 300 consecutive patients from a tertiary care urology clinic at Colombo South Teaching Hospital, Sri Lanka. A self-administered questionnaire was used. Chi-Square test was used for statistical analysis.
\end{abstract}

The mean age of the patients was 55.9 (range:11-85). Eight patients (2.7\%) had previous known hearing impairment. Repetition of instructions were required at least once due to hearing impairment in 27.3\% (once: $14.3 \%$; twice: $7.0 \%$; three times:3.3\%; $>$ three times:2.7\%). However, around 81.7\% reported that they had no hearing problems while others reported some difficulty (18.3\%: mild $-10.3 \%, \mathrm{n}=31$; moderate $-4.3 \%, \mathrm{n}=13$ and severe-3.7\%,n=11). Nine patients $(3.0 \%)$ required lowering of the mask to enable lip reading in order to understand the instructions and all reported moderate/severe hearing impairment (moderate: $\mathrm{n}=1$, severe: $\mathrm{n}=8$ ). Patient reported hearing impairment was significantly higher in those aged $\geq 65$ years $(31 \%$ vs. $11 \%$, p $<0.001)$.
Around 18\% of our cohort experienced some form of hearing difficulty. Thus, awareness among the healthcare providers and general public should be improved. Further studies on the impact of this problem with special focus on possible solutions are necessary.

Key words: COVID - 19, SARS Co-2 virus, communicating difficulties, face masks

\section{Introduction}

The COVID-19 pandemic has caused many challenges to the country's economy and the livelihood and socio-cultural beliefs of general public worldwide and in Sri Lanka (1-3). The COVID-19 has posed significant impact to health care systems and health care workers altering work pattern and burden $(3,4)$. The impact is not only due to the illness itself but also due to measures recommended and imposed to contain the pandemic $(5,6)$. Patients and health care personnel find it difficult to communicate with each other due to wearing masks and social distancing (8). The difficulties are more among those with hearing impairment and in health care facilities with lot of background noise due to overcrowding. In USA, more than $70 \%$ of those aged above 65 years have some form of hearing impairment (7). Approximately $20 \%$ of urban population in Sri Lanka have hearing impairment and of them, $86 \%$ 
had not sought any medical treatment (8). In Sri Lanka, a considerable proportion of those seeking treatment for their illnesses are elderly patients and clinics of state hospitals are overcrowded with limited space. Furthermore, some health care facilities allow only the patient to meet the doctor to save space in order to ensure social distancing. Therefore, it is likely the current pandemic causes hardships to those with hearing impairment in Sri Lanka. We aimed to provide a preliminary description about difficulties faced by a group of patients due to hearing impairment. Providing robust statistical analyses or giving objective quantification of the current burden is beyond the scope of the analysis.

\section{Methods}

We collected data from 300 consecutive patients from a tertiary care urology clinic at Colombo South Teaching Hospital, Sri Lanka from 1 December 2020 to 31 December 2021. A self-administered questionnaire was used to collect details on basic demography and subjective hearing assessment to determine the extent of the problems encountered by patients due to inadequate hearing of speech secondary to face masks and social distancing. The study was approved by the Ethics Review Committee of the institute. Chi-Square test was used for statistical analysis. A p value of less than 0.05 was considered statistically significant.

\section{Results}

The mean age of the patients was $55.9(\mathrm{SD}=16.8$, range: $11-85)$ years. Around $36.7 \%(n=110)$ were aged 65 years or more. Majority of the patients were under investigation or treatment for bladder outflow obstruction $(\mathrm{n}=91,30.3 \%)$, urolithiasis $(\mathrm{n}=96,32 \%)$, urinary tract infection $(\mathrm{n}=22,7.3 \%)$ and prostate carcinoma $(n=21,7.0 \%)$.

Around $27.3 \%$ of them required repetition of $-34-$ instructions at least once during the clinic visit (once: $14.3 \%, \mathrm{n}=43$; twice: $7.0 \%, \mathrm{n}=21$; three times: $3.3 \%, \mathrm{n}=10$; and more than three times $2.7 \%, n=8)$. Fifty-five patients $(18.3 \%)$ admitted experiencing some form of hearing difficulty (mild-10.3\%, $\mathrm{n}=31$; moderate- $4.3 \%, \mathrm{n}=13$ and severe-3.7\%, $\mathrm{n}=11)$. Nine patients $(3.0 \%)$ required lowering of the mask by the doctor allowing them to lip-read in order to understand the instructions and all of them had moderate to severe hearing impairment (moderate: $\mathrm{n}=1$, severe: $\mathrm{n}=8$ ); 5 had history of hearing impairment. Interestingly, only 8 patients $(2.7 \%)$ had previous known hearing impairment. As expected, patients experiencing difficulties in comprehending doctor's instructions was significantly higher among those older than 65 years $(31 \%$ vs. $11 \%, \mathrm{p}<0.001)$.

\section{Discussion}

Our preliminary study showed that around $18 \%$ of the cohort experienced some form of hearing difficulty. Several reasons could be postulated for the observed results. Important cues such as lip movements and facial expressions are hampered by wearing masks while reducing the volume and clarity of the sound. The use of a face shield in addition to the mask further dampens the sound. Thus, individuals with hearing impairment may face difficulty in understanding the important facts during communication both in the health care facilities as well as in the community. Disrupting interpersonal communication in general may lead to social isolation and withdrawal causing loneliness and depression (9). However, the relevance of these negative effects is not sufficiently emphasised to the health care workers. The actual impact on patients' health care due to poor hearing and lack of communication secondary to COVID pandemic restrictions has not been well documented. A preliminary study conducted in a tertiary care centre in Italy including 59 patients 
showed that around $25 \%, 37 \%$ and $24 \%$ of patients faced mild, moderate and severe difficulties in hearing respectively (9). Sound attenuation (44\%) and inability to lip read (56\%) were the common reasons for poor hearing.

Although hearing impairment due to COVID -19 disease is described and drawn lot of attention, it is rare. The difficulties in hearing due to face masks and social distancing is common and much more important to patients. Patients may not understand medical advice properly leading to unnecessary difficulties and morbidity. As clinicians, we should be mindful of the potential negative consequences of face masks and social distancing. The health care workers and also the general public should be made aware of the psychosocial problems faced by those with difficulties in hearing amidst COVID pandemic restrictions. Such awareness would enable the health care workers to identify such patients and take simple steps like speaking loudly, reducing the distance to the minimum possible, allowing precautionary lip reading and providing less noisy environments. A carer may be allowed to accompany the patient and important instructions can be given in writing to the patient. There are more sophisticated measures that can be devised to overcome this problem. Face masks with a transparent area around the mouth may allow lip reading, while providing necessary protection against aerosolised particles. When wearing face shields, an inbuilt microphone may be utilised to amplify the sound of the speaker. If facilities permit, for selected patients a separate cubicle may be set up with reduced background noise and a microphone while having a transparent partition between the health care worker and the patient to minimise transmission of the virus. Digital technology may be useful, such as applications that convert speech to text (10). With the implementation of effective COVID-19 vaccination, some restrictions of social Vol.33, No.1, July 2021 distancing may be loosened to overcome this problem (11).

Our study is a small prospective study with methodological limitations such as lack of objective assessment of hearing impairment or use of validated instruments. Despite these methodological limitations, we believe this preliminary study provides a useful overview of the burden due to difficulties faced by hearing impairment for clinical consideration (12).

\section{Conclusion}

Data on the prevalence and the burden due to difficulties faced by the hearing-impaired patients is limited, especially in the local setting during the COVID pandemic. The findings of this preliminary study show that this problem may be common and should be given adequate emphasis. Thus, awareness among the healthcare providers and the general public should be improved. Further studies on the impact of this problem with special focus on possible solutions are necessary.

Conflict of interest: All authors declare that they have no conflicts of interest.

\section{References}

1. Jayasinghe $R$, Ranasinghe $S$, Jayarajah $U$, Seneviratne S. Quality of online information for the general public on COVID-19. Patient Education and Counseling. 2020;103(12):25947.

2. Borges do Nascimento IJ, Cacic N, Abdulazeem HM, von Groote TC, Jayarajah U, Weerasekara I, et al. Novel coronavirus infection (COVID-19) in humans: a scoping review and meta-analysis. Journal of clinical medicine. 2020;9(4):941.

3. Jayasinghe R, Jayarajah U, Seneviratne S. Consensus on peri-operative surgical practice 
during the COVID-19 pandemic: an appraisal of the literature. Sri Lanka Journal of Surgery. 2020;38(2):57-61.

4. Jayasinghe R, Welikala N, Gunaratne R, Jayarajah U, Dassanayake V, Seneviratne S. Anaesthetic implications during the COVID-19 pandemic: an appraisal of the literature. Sri Lanka Journal of Surgery. 2021;39(1):48-52.

5. Wijerathne PK, Nanayakkara K, Basnayake O, Gunapala N, Jayarajah U. Impact of COVID-19 on postgraduate surgical training: the trainees' perspective. Sri Lanka Journal of Surgery. 2020;38(3):53-5.

6. Borges do Nascimento IJ, von Groote TC, O’Mathúna DP, Abdulazeem HM, Henderson C, Jayarajah U, et al. Clinical, laboratory and radiological characteristics and outcomes of novel coronavirus (SARS-CoV-2) infection in humans: Asystematic review and series of metaanalyses. PloS one. 2020;15(9):e0239235.

7. West JS, Franck KH, Welling DB. Providing health care to patients with hearing loss during COVID-19 and physical distancing. Laryngoscope Investigative Otolaryngology. 2020;5(3):396-8.

8. Nugegoda D, Balasuriya S. Health and social status of an elderly urban population in Sri Lanka. Social Science \& Medicine. 1995;40(4):437-42.

9. Park J. Unraveling the Invisible but Harmful Impact of COVID-19 on Deaf Older Adults and Older Adults with Hearing Loss. Journal of gerontological social work. 2020:1-4.

10. Trecca EMC, Gelardi M, Cassano M. COVID-19 and hearing difficulties. Am J Otolaryngol. 2020;41(4):102496-.

11. Seneviratne SL, Jayarajah U, Abeysuriya V, Rahman A, Wanigasuriya K. COVID-19 Vaccine Landscape. Journal of the Ceylon College of Physicians. 2020;51:120-31.

12. Gunaratne A, Jayarajah U, Abeygunasekera A. Continuing metamorphosis of evidence-based medicine: the past, present and the future. Galle Medical Journal. 2020;25(4):118-21. 